
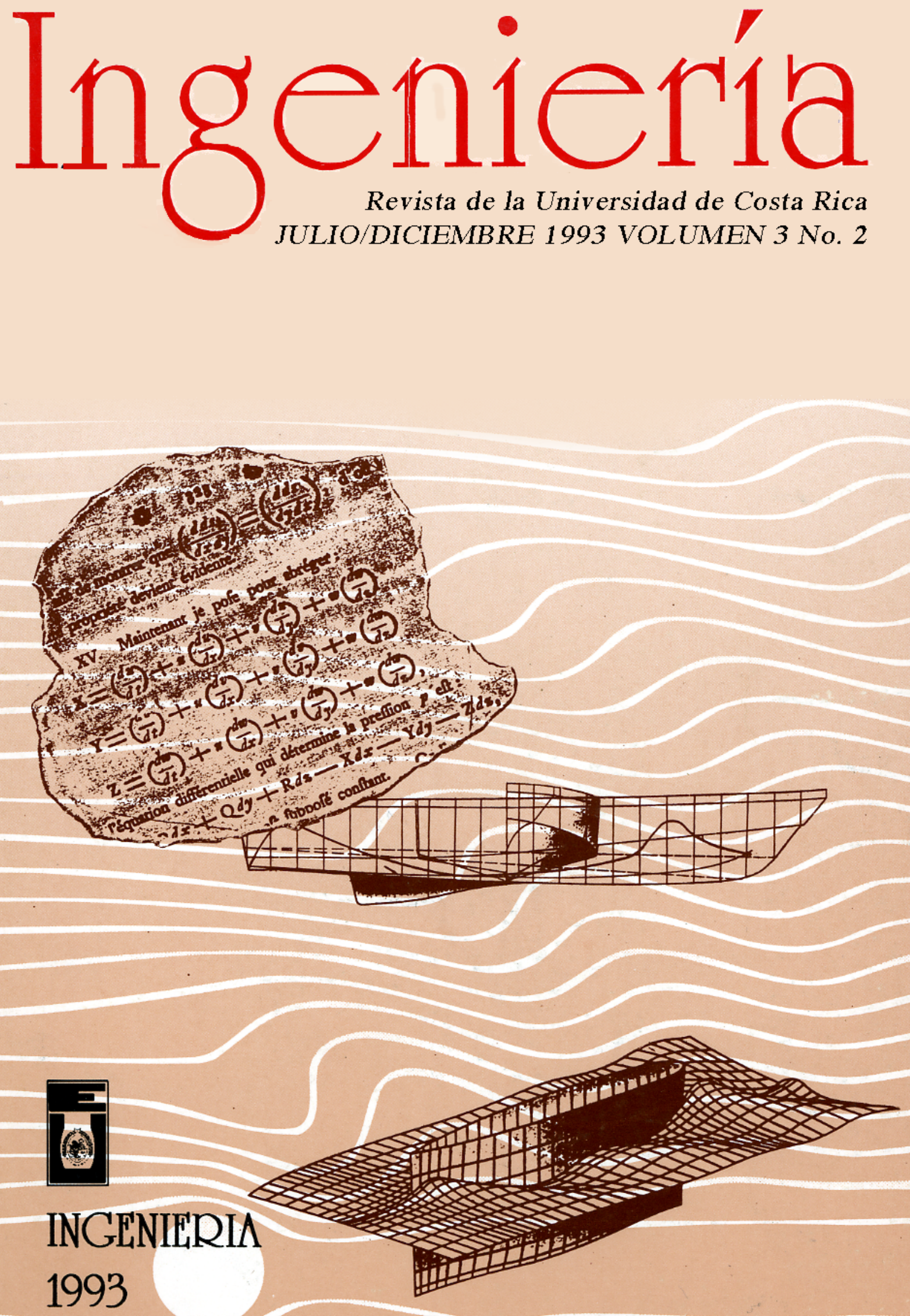


\title{
INGENIERIA DIDACTICA
}

\author{
Ing. Fernando Cajas Dominguez*
}

\section{Resumen}

\begin{abstract}
Se revela el carácter tecnológico de las "Ciencias de la Educación" a partir de una concepción de tecnología no reducida a las ingenierías tradicionales. Se dilucida el concepto de práctica educativa y sistema educativo apoyandose en la Teoría General de Sistemas y en las funciones de estado. Se analizan las consecuencias del enfoque tecnologico para una posible teoría de la educación.
\end{abstract}

\section{Summary}

The technological character of the "sciencie education" is shown as a concept of technology not reduced to traditional engineering. The educational practice and educational system are defined with the help of the General System Theory and funtions of state. The consequences of the technological approach are analysed.

\section{INTRODUCCION}

Estamos acostumbrados a identificar la práctica de la ingeniería con procesos industriales o con sus vecinos cercanos y francamente resulta difícil imaginarse la existencia de un enfoque tecnológico en los procesos educativos, a no ser por una identificación de tecnología educativa con artefactos, aparatos audiovisuales que nos ayudan en la tarea de la docencia. Esto posee varias explicaciones, veamos dos, las que consideramos las más importantes.

Explicación 1. Una concepción de tecnología restringida a artefactos, en el peor de los casos y a las ingenierías tradicionales (ingeniería civil por ejemplo) en el mejor, no permite visualizar la amplitud del enfoque tecnológico.

Profesor titular de la Universidad de San Carlos de Guatemala, Carrera de Ingeniería, Centro Universitario de Occidente (CUNOC), Quetzaltenango, Guatemala, C.A.
Explicación 2. La naturaleza conceptual de las denominadas "Ciencias de la Educación", en donde el planteamiento de los problemas educativos suele hacerse con el mínimo de herramientas conceptuales y en lenguaje ordinario, no permiten encontrar algún parecido con la manera en que los ingenieros resuelven los problemas.

Para poder analizar el posible carácter tecnológico de las "Ciencias de la Educación" es preciso disponer de una marco de referencia claro y coherente con la ciencia y la tecnología moderna. Dicho marco lo hemos desarrollado parcialmente en este trabajo, pero la mayoría se encuentra en otra parte (Bunge 1980, 1983, 1985a, 1985b, 1989; Cajas 1991, 1992a, 1992b; Herrera 1989, 1990, 1991a, 1991b, 1991c, 1992).

\section{LA PRACTICA EDUCATIVA}

La palabra educación posee muchos significados, pero existe cierto consenso en creer que "la 
educación pertenece a la categoría de la acción" (Mialaret 1981), en ese sentido nosotros la concebimos como una práctica social (Althusser 1988), que se da en un sistema concreto y que posee un sistema conceptual (Bertalanffy 1976). Tomaremos prestado de nuestro marco de referencia el concepto de práctica social, junto con algunos postulados que permitirán dilucidar el concepto de práctica educativa.

Definición 1. Práctica Social (P): es una actividad orientada de los hombres organizados en subsistemas sociales, de tal manera que ella cámbia un objeto en alguna cosa, artefacto o proceso usando ciertos medios de producción. Es una forma de apropiación biunívoca entre el hombre y el ambiente (apropiación de la naturaleza) y entre el hombre y el hombre (apropiación de organización). (Herrera 1991a, 54-55).

Postulado 1. Toda práctica social $\mathbf{P}$ tiene una "estructura genérica de producción", compuesta por cuatro elementos: fuerza de trabajo humano $\mathbf{W}$, medios de producción $\mathbf{m}$, el objeto de transformación $\vartheta$, y el producto final p. (Herrera 1991a, 58). En forma breve:

$$
\mathbf{P} \hat{=}<\mathbf{W}, \mathbf{m}, \vartheta, \mathbf{p}\rangle
$$

donde el símbolo semántico $\triangleq$ se lee: "se representa por" o "se modela por".

Postulado 2. Toda práctica social se puede enfocar como trabajo abstracto creador de valor intrínseco y trabajo concreto de valor de uso (lo que incluye a las prácticas teóricas) (Herrera 1991a, 56).

Si queremos aprovechar la riqueza que provee el concepto de práctica social, es preciso que pensemos que los seres humanos no solo transformamos materiales físicos, sino también estados mentales (condicionando por ejemplo). Con esta aclaración podemos presentar la siguiente definición:

\section{Definición 3. Práctica Educativa (PE):}

Práctica social cuyo objetivo es la producción de aprendizaje de los componentes sociales.

Los conceptos claves de la definición anterior son: práctica social y aprendizaje. Como no es posible definir todos y cada uno de nuestros conceptos, es preciso adoptar de nuestro marco de referencia (marco teórico) dichos conceptos, tal como hemos hecho con el de práctica social, así el concepto de aprendizaje debe tomarse de la psicología moderna ${ }^{(1)}$.

Caracterizamos más precisamente la PE utilizando el postulado 2, pues como práctica social puede existir una Práctica Educativa EmpíricaConcreta (PEE) - ej.: la que hace el profesor en aula y una Práctica Educativa Teórico-Conceptual (PEC) -ej.: la que hace el investigador de la educación-, así:

\section{Definición 5. PE = PEE $\cup$ PEC}

Ilustraremos la práctica educativa empíricoconcreta (PEE) utilizando el postulado 2.

W: en la PEE está representado por el educador E.

m: en la PEE está representado por el currículum $\mathbf{C}$ en el sentido ámplio de la literatura pedagógica ${ }^{(2)}$.

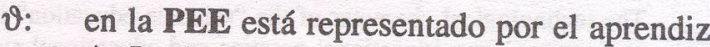
A. Lo que en realidad interesa es el estado

1 Utilizaremos el siguiente concepto de aprendizaje: "Sea $\mathbf{E}$ una clase de procesos o sucesos en un sistema neural (que incluye algún subsistema plástico) de un animal a, y sea $\mathrm{S}$ la clase de estímulos (externos 0 internos) que a puede sentir o detectar. Entonces, decimos que a ha aprendido e $\epsilon E$ en presencia de $s \varepsilon S$ durante el intervalo de tiempo $\left(t_{1}, t_{2}\right)$ si y solo si:

1) antes de $t_{1}$ cuando a se encontraba en presencia de e, en a no aparecía e.

2) después de $t_{2}$ cada vez que a siente $s$ aparece también e (es decir a memorizó s) (Bunge 1985a, 157).

En la literatura pedagógica existe cierta uniformidad en considerar el currículum como el contenido que debe llegar a dominar el estudiante, así como las políticas y procedimientos institucionales empleados para lograrlo (Hilda Taba). Nos interesa una visión aún más amplia de currículum, como la que se presenta en Moreira y Axt (1991), que es una adaptación del modelo de Johnson, el cual incluye la cultura como punto de partida, los criterios de estructuración y selección, los valores y metas de la sociedad, el sistema instruccional, la evaluación. Esta visión es tan amplia que no se restringe a la enseñanza de la ciencia, por lo que se presenta en todas las prácticas educativas empíricas concretas. 
mental ${ }^{(3)}$ de $\mathbf{A}$ al tiempo $t_{1}$, dicho estado le denominaremos $\mathrm{S}_{1}$.

p: en la PEE está representado por el aprendiz A. Lo que en realidad interesa es el estado mental de $\mathbf{A}$ al tiempo $t_{2}$, tal que $t_{2}>t_{1}$, dicho estado lo denominaremos $\mathrm{S}_{2}$.

En forma breve podemos representar la estructura de la práctica educativa de la forma siguiente:

$$
\mathrm{PEE} \stackrel{\wedge}{=} \mathbf{E}, \mathbf{C}, \mathbf{A}\rangle
$$

\section{EL CARACTER TECNOLOGICO DE LAS CIENCIAS DE LA EDUCACION}

Como hemos visto, la esencia de la educación está en el concepto de acción (acción con propósito), la acción del educador sobre el aprendiz con el propósito de producir aprendizaje, esto es, un cambio de estado mental (Mialaret 1981, 9; Bunge 1985a 92, 157). Las consecuencias de este planteamiento son:

1) $\mathrm{El}$ educador $\mathrm{E}$ actúa de manera intencional, es decir de manera consciente.

2) El educador $\mathbf{E}$ actúa de manera sistemática.

Para realizar las dos actuaciones anteriores, el educador $\mathbf{E}$ debe hacerse una representación anticipada del estado final al que desea llevar al aprendiz A. Dicho en otras palabras el educador debe realizar un diseño.

Definición 6. Diseño: Representación anticipada de un objeto (cosa, estado o proceso) con ayuda de algún conocimiento (Bunge 1989; Herrera 1989,1991 ).

Estado mental. Intuitivamente podemos pensar en el listado de propiedades que caracterizan a la mente en un momento dado. El problema de esta intuición es que hay que definir "mente", para ello seremos coherentes con la neurociencia, así: "Más precisamente supondremos que cualquier estado o proceso mental es un estado de actividad o proceso de algún sistema neural no comprometido (plástico), sin contar sus funciones domésticas". (Bunge 1985a, 92).
Este diseño se presenta en la práctica educativa desde hace mucho tiempo y lo que ha variado es el conocimiento que sustenta al diseño, en particular la época actual se caracteriza por una intensa racionalización de los procesos de transformación, no solo en la explicación racional del mundo sino en la optimización de las acciones (Herrera 1989). Esta peculiaridad de la modernidad puede conceptualizarse a través de la idea de práctica tecnológica.

\section{Definición 7. Práctica tenológica (PT):}

Práctica Social cuyo objetivo inmediato es la transformación y el control de cosas y sistemas (naturales, sociales o formales) mediante acciones racionales basadas en el conocimiento científico existente (Herrera 1991a, 59).

Existe cierto consenso en aceptar que la práctica educativa es una práctica social (Althusser 1988; Ordóñez 1991), pero de esto a que sea una práctica tecnológica, hay diferencia ${ }^{(4)}$. El carácter tecnológico de la práctica educativa se visualiza a partir de que el objetivo inmediato de la misma es:

\section{i) Transformar el estado mental del aprendiz A}

Esta transformación puede hacerse con ayuda de distintos tipos de conocimiento, tradicionalmente el diseño de los educadores está basado en cierto cuerpo de conocimiento empírico. Sin embargo, esto parece estar cambiando trabajos recientes en investigación, en enseñanza de la ciencia muestran que se empiezan a encarar científica y sistemáticamente algunos problemas educativos. Dicho de otra forma: la educación ha tomado el camino del método de la ciencia (no como receta), así en ciertas secciones de la educación moderna la transformación del estado mental de aprendiz se basa en:

\section{ii) Conocimiento Científico}

Estas dos características (i y ii) son propias de la racionalidad práctica que caracteriza la tecnología moderna (Bunge 1983, 1985a, 1985b; Herrera 1989, 1990, 1991a, 1991b). En efecto, el

En Bunge (1985, 1989); Cajas (1992a); Carrasco et al. (1984); Herrera $(1989,1992)$; se presenta de manera explícita el carácter tecnológico de la educación. 
planteamiento de la problemática educativa actual tiende a realizarse desde una perspectiva tecnológica, así Ausbel explica: "Pocos son los que contradicen que la educación es una ciencia aplicada o de ingeniería" (Ausbel 1976, 30).

\section{UN EJEMPLO PARTICULAR: LA EDUCACION MATEMATICA}

Partimos de la idea de que la educación matemática no es más que un tipo particular de "ciencia de la educación". En ese sentido debe compartir el carácter tecnológico analizado en la sección anterior; sin embargo, en esta parte seremos más puntuales. En efecto, la siguiente cita puede ayudarnos a clarificar dicho carácter. "La Educación Matemática enfrenta dos compromisos fundamentales:

a) Dar solución a corto plazo a las demandas educativas de una población creciente en número y complejidad, y

b) desarrollar un cuerpo teórico de conocimiento que, a largo plazo, sustente soluciones más permanentes" (Waldegg 1989, 55).

Con estas características la educación matemática cumple la definición 7 , por lo que es una práctica tecnológica. Realizaremos una comparación entre una tecnología clásica y una tecnología social. El cuadro siguiente precisa esto.

\section{Cuadro No. 1}

\begin{tabular}{|c|c|c|c|}
\hline Protociencia: & Ciencia: & $\begin{array}{l}\text { Tecnología } \\
\text { Genérica: }\end{array}$ & Tecnología: \\
\hline $\begin{array}{l}\text { Alquimia, } \\
\text { Mineralogía } \\
\text { Antigua. }\end{array}$ & Química & $\begin{array}{l}\text { Tecnología } \\
\text { clásica }\end{array}$ & $\begin{array}{l}\text { Ing. Quími- } \\
\text { ca }\end{array}$ \\
\hline $\begin{array}{l}\text { Enseñanza } \\
\text { como arte: } \\
\text { Grecia Antigua, } \\
\text { Medioevo, con- } \\
\text { temporaneo }\end{array}$ & $\begin{array}{l}\text { Sociología } \\
\text { Psicología } \\
\text { Matemática }\end{array}$ & $\begin{array}{l}\text { Tecnología } \\
\text { social }\end{array}$ & $\begin{array}{l}\text { Educación } \\
\text { Matemática }\end{array}$ \\
\hline
\end{tabular}

El Cuadro No.1 sigue la estructura de uno presentado en Bunge (1985b, 210). El nuestro tiene la peculiaridad de referirse explícitamente a la educación matemática y en él introducimos el concepto de tecnología genérica, la cual puede ser clásica (civil, por ejemplo) o social. Para hacer más explícita la comparación propongamos dos problemas por resolver, uno en el contexto de la ingeniería química (problema 1) y otro en el de la educación matemática (problema 2).

Problema 1: Calentar un fluido de 200,000 gph de petróleo A, de $100^{\circ} \mathrm{F}$ a $200^{\circ} \mathrm{F}$ (Peters and Timmerhaus 1968, 786).

Problema 2: Mejorar el proceso enseñanza/aprendizaje del álgebra de la escuela secundaria (Rojano y Filloy 1989).

En ambos podemos detectar que:

a) Algo debe ser seguido, ya diseñar un intercambiador de calor para resolver el problema 1 ó diseñar un plan para profesores de álgebra, con el fin de resolver el problema 2.

b) El diseño para resolver ambos problemas puede estar basado en teorías tecnológicas, esto es: casos particulares de teorías científicas o en estudios experimentales guiados por el método de la ciencia. discutida.

El siguiente cuadro resume la analogía Cuadro No. 2

Ingeniería Química

Educación Matemática
Diseño de: un intercambio de calor.

Planta petroquímica

Termodinámica clásica

Teoría Tecnológica: transferencia de calor, coeficientes de calor, estudios de laboratorio

diseño práctico
Diseño de: un plan para enseñanza del álgebra

Sistema educativo concreto

Teoría de aprendizaje

Teoría teconológica:

Psicología educativa Ausbel Modelos teóricos locales Estudios experimentales
Hay que destacar la importancia de la analogía, pero no se puede exagerar. Aunque la educación matemática comparte con la ingeniería clásica un enfoque utilitarista, la problemática de la educación matemática parece ser más compleja, la 
razón es que se refiere a más niveles, lo cual implica manejar más variables.

Para terminar analizaremos las posibilidades reales de un planteamiento tecnológico en educación, para ello es preciso definir el concepto de sistema educativo dado que es allí donde se dá la práctica educativa, ese trabajo lo haremos en la siguiente sección.

\section{SISTEMAS CONCRETOS DE LA EDUCACION}

Suponemos que una sociedad humana es analizable en cuatro subsistemas básicos, a saber: el económico $\mathrm{E}_{\mathrm{E}}$, el biológico $\mathrm{E}_{\mathrm{B}}$, el político $\mathrm{E}_{\mathbf{P}} \mathrm{y}$ el cultural $\mathrm{E}_{\mathrm{C}}$ (Bunge 1980, Herrera 1991b). Utilizaremos los conceptos básicos de la teoría general de sistemas (Bertalanffy 1976, Bunge 1985b, Herrera 1991c). Con estas aclaraciones podemos plantear la siguiente definición.

Definición 8. Sea $\mathbb{E}$ un sistema concreto, diremos que $\mathbb{E} \in \mathbb{E}_{\mathrm{C}}$ es un sistema educativo si y solo si:

a) La composición C ( $\mathbb{E}$ ) está formada por educadores, aprendices, administradores, empleados educativos;

b) $\mathrm{El}$ ambiente directo $\mathrm{A}(\mathbb{E})$ está formado por libros, computadoras, salones de clases, salones de laboratorio, bibliotecas, salones de administración, etc.;

c) La estructura de $\mathrm{E}(\mathbb{E})$ es el conjunto de relaciones sociales, en especial la práctica educativa.

El introducir el concepto de sistema educativo en un contexto teórico tiene ventajas, una de la más importantes es que podemos representarlo, esto es hacernos un modelo conceptual del mismo. Para ello pensemos en un sistema educativo elemental al que denominaremos "el menor de los sistemas educativos" cuyas características son las siguientes:

a) La composición de $\varepsilon$, esto es $C$ ( $\varepsilon$ ) es un educador y un aprendiz, es decir: $\mathrm{C}(\varepsilon)=$ $\{\mathbf{E}, \mathbf{A}\}$ b) El ambiente de $\mathbb{E}$ está contenido en el ambiente de $\varepsilon$, donde $\mathbb{E}$ es el sistema educativo dado por la definición 8 , esto es:

$$
\mathrm{A}(\mathrm{E}) \subset \mathrm{A}(\varepsilon)
$$

c) La estructura de $\varepsilon$ está contenida en la estructura de $[\mathrm{E}$, simbólicamente:

$$
\mathrm{E}(\varepsilon) \subset \mathrm{E}(\mathbb{E})
$$

d) El objetivo de es producir un aprendizaje en $\mathbf{A}$.

Este sistema educativo elemental $\varepsilon$ se puede representar por medio de la función $\mathrm{F}$, que llamaremos función de estado y que por séncillez podemos suponer que tiene un número finito de componentes, es decir:

$$
F=\left\langle f_{1}, f_{2}, \ldots, f_{n}\right\rangle
$$

donde cada $f_{i}(i=1,2, \ldots n)$ representa propiedad i del sistema $(\varepsilon)$, de un total de $n$ propiedades que lo determinan. En el caso del sistema educativo las propiedades que lo determinan son de múltiples niveles, a saber: afectan factores psicológicos, sociológicos, etc., así que lo único promisorio es un enfoque multidisciplinario.

Si estamos interesados en propiedades especiales globales del sistema y no en una localización precisa del mismo, podemos suponer que $\mathrm{F}$ es una función dependiente del tiempo, suyos valores son n-tuplas de números reales (Bunge 1985, 86-87), es decir podemos suponer que:

$$
\mathrm{F}: \mathrm{T} \rightarrow \mathbb{R}^{\mathrm{n}}
$$

donde $T$ es el tiempo y $\mathbb{R}$ el conjunto de los números reales.

Ahora es preciso introducir el concepto de estado de un sistema. Intuitivamente podemos pensar en el conjunto de propiedades, relaciones y situaciones que en un momento dado constituyen el sistema. En el caso particular del sistema educativo elemental podemos ser más formales y decir:

Definición 9. Estado del sistema educativo $\varepsilon$ : el estado $\mathbf{S}$ del sistema educativo $\varepsilon$ en el tiempo $t \in T$ es $S=F(t)$. 
El estado $\mathrm{S}$ al tiempo $\mathrm{t}$ se puede representar por medio de un punto en espacio cartesiano n-dimensional. Sin embargo, no todos los puntos del espacio son estados posibles, existen limitaciones dadas por las leyes del sistema.

Sea $S_{1}$ el estado de $\varepsilon$ al tiempo $t_{1}$ y $S_{2}$ el estado del mismo sistema al tiempo $t_{2}$, tal que $t_{1}<$ 々. El cámbio de estado del sistema educativo se puede representar por medio de:

$$
\Delta S=S_{2}-S_{1}
$$

El cambio de estado $\Delta S$ describe cuando el sistema va de un punto a otro, pero no explica cómo lo hace. Para averiguar la dinámica de $\Delta \mathrm{S}$ es preciso conocer IF. Esta tarea es muy difícil, la razón es que aún desconocemos las leyes que deben formar $F$. Sin embargo, podemos esbozar algunos niveles de las distintas $\mathrm{f}_{\mathrm{i}}$ que pertenece a IF, congruentes con la investigación actual en educación, veamos un listado al azar:

$f_{1}$ : factores psicológicos,

$\mathrm{f}_{2}$ : factores del currículum,

$f_{3}$ : factores sociales, ideológicos

$\mathbf{f}_{4}$ : factores biológicos,

$f_{5}$ : factores filosóficos, etc.

o sea que no queda determinado solamente por factores psicológicos, sino por mucho más, lo que explica la incapacidad de las teorías de aprendizaje (psicològicas) para explicar los procesos educativos.

Para terminar ilustraremos las posibilidades de teorización en educación.

\section{POSIBILIDADES DE TEORIZACION}

Muchas de las investigaciones actuales en educación tienen sus raices teóricas en la psicología educativa de Ausbel (Novak 1991, Moreira 1991). La suposición más importante de la teoría ausbeliana es la siguiente: "Si tuviese que reducir toda la psicología educativa a un solo principio, enunciaría éste: de todos los factores que influyen en el aprendizaje, el más importante consiste en lo que el alumno ya sabe. Averigüese esto y enséñese consecuentemente" (Ausbel 1976, 78) Dicho en términos del lenguaje que implementamos en la sección anterior, podemos decir que:

$$
S_{2}=\mathbb{F}\left(S_{1}\right)
$$

fórmula que se lee: el estado final $S_{2}$ es una función del estado inicial $S_{1}$. Donde $S_{1}$ y $S_{2}$, son estados del sistema educativo del aprendiz A, ya definidos.

En el caso de la teoría ausbelina, la función IF posee tres componentes, que son:

$$
\text { IF }=\left\langle\mathrm{f}_{1}, \mathrm{f}_{2}, \mathrm{f}_{3}\right\rangle
$$

donde, $f_{1}$ : factores emotivos, esto es si el alumno quiere o no;

$\mathrm{f}_{2}$ : factores cognoscitivos, si el alumno posee la estructura cognoscitiva adecuada o no;

$\mathrm{f}_{3}$ : si el contenido a enseñar es lógicamente coherente.

Ilustraremos el cámbio de estado mental que persigue la PEE utilizando la teoría de Ausbel junto al andamiaje sistémico que le hemos colocado (la descripción de la teoría no es el propósito del artículo y la adaptación formal de las funciones de estado a dicha teoría es un problema abierto). Como solo es posible dibujar en dos dimensiones, ilustraremos el cámbio de estado con dos propiedades psicológicas de la mencionada teoría, esto es factores emotivos $\left(f_{1}\right)$ y factores cognoscitivos $\left(f_{2}\right) \cdot S_{1}$ y $S_{2}$, tienen el mismo significado que en la definición de PEE.

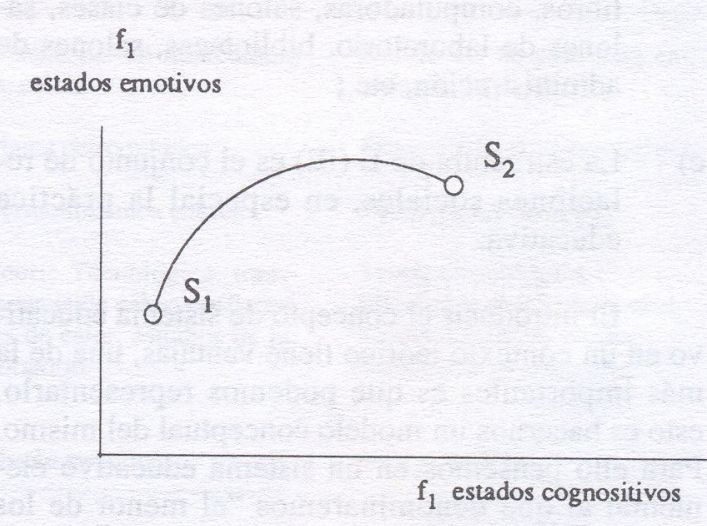

Figura 1. Proyección dimensional del espacio de estados mentales como posible producto de la práctica educativa empírico concreta (PEE). El arco de curva representa un proceso mental entre una multitud de posibilidades. 
al $S_{2}$ es una fun$S_{1}$ y $S_{2}$, son estaendiz $\mathbf{A}$, ya defi-

elina, la función 㲅:

o es si el alumno

os, si el alumno gnoscitiva adeseñar es lógicatado mental que oría de Ausbel hemos colocaes el propósito 11 de las funcioproblema abieren dos dimenestado con dos ncionada teoria, tores cognoscisignificado que

dos cognositivos

io de estados meneducativa empírico un proceso mental
Como puede verse, para ir de $S_{1}$ a $S_{2}$ existen muchos caminos, pero no todos son igualmente eficientes, es tarea de las ciencias de la educación sugerir el camino más eficiente, de ahí su carácter tecnológico.

\section{COMENTARIOS FINALES}

En resumidas cuentas, es posible esperar en un futuro próximo una verdadera ingeniería didáctica, para ello los educadores deben aceptar una reestructuración del lenguaje con que suelen plantear los problemas educativos, el cual debe ser cambiado por un lenguaje preciso. Esto no bastará, es vital buscar pautas de comportamiento, leyes para llenar F. Toda esta fundamentación es necesaria para optimizar la actividad de la educación.

\section{BIBLIOGRAFIA}

Althusser, L. La revolución teórica de Marx. Siglo XXI: México. 1988.

Ausbel, D. Psicologia educativa. Trillas, México. 1976.

Bertalanffy, L. (1976) Teoría general de los sistemas. Fondo de cultura económica, México.

Bunge, M. (1980): Ciencia y Desarrollo. Siglo XXI. Buenos Aires.

(1983): La investigación Científica. Ariel, Barcelona

(1985a): El problema mente-cerebro. Tecnos, Madrid.

(1985b): Epistemología. Ariel, Barcelona.

(1989): Seudociencia e ideología. Alianza Universidad, Madrid.

Cajas, F. (1991): "Aquiles y la tortuga: un problema filosófico" Cuarto congreso Centroamericano y del Caribe de Historia de la ciencia y la tecnología, Universidad de Costa Rica.

1992a): "Matemática educativa: Una ingeniería didáctica" Primer congreso nacio- nal de matemática educativa, Universidad de Panamá.

(1992b): "Supuestos geométricos de una teoria fisica elemental" Primer congreso nacional de matemática educativa, Universidad de Panamá.

Carrasco et al. (1984): Teoría de la educación. Anaya, Madrid.

Herrrera, R. (1989): "La práctica tecnológica" Rev. Filosofía Universidad de Costa Rica XXVII (66), 349-359

(1990): "Tecnologia y sociedad" Rev. Filosofia, Unive. Costa Rica, XXVIII (67/68), $77-84$.

(1991a): "Sistemas conceptuales de la Tecnología” Rev. Ingeniería, 1(1): 67-78, San José Costa Rica.

(1991b): "Cultura General y Especialidad" Tercer congreso centroamerica-no y del Caribe de Historia de la Ciencia y la tecnología, Univ. Costa Rica: San José

(1991b): "Tecnología: un marco teórico" Mem. cuarto cong. Centroamericano y del Caribe de Historia de la Ciencia y la Tecnología. Univ. Costa Rica: San José.

Mialaret, G. (1981): Ciencias de la educación. Oikos-ta, Barcelona.

Novak, J. (1991): "Ayudar a los alumnos a aprender. La oponión de un profesor-investigador" Enseñanza de las ciencias, 9(3), 215-228.

Peters, M. and Timmerhaus, (1967): Plant design and economics for chemical engineers. Macgraw-Hill, Tokyo.

Rojano, T. y Filloy, E. (1989): "Cursos para profesores basados en investigaciones recientes en didáctica del álgebra". Cuadernos de investigación, No.12, Año III, 54-59.

Waldegg, G. (1989): "Evaluación del trabajo académico en matemática educativa Avance y perspectiva, No.39, 53-56. 